ISAHP 2005, Honolulu, Hawaii, July 8-10, 2005

\title{
MEASURING EFFICIENCY OF PRODUCTION UNITS BY AHP MODELS
}

\author{
Josef Jablonsky \\ Department of Econometrics, University of Economics \\ Praha, 13067 Czech Republic \\ jablon@vse.cz, URL: http://nb.vse.cz/ jablon/
}

Keywords: AHP, data envelopment analysis, multiple criteria decision making, efficiency

Summary: The paper deals with models and methods for evaluation of efficiency of production units. The standard modeling approach for evaluation of efficiency is data envelopment analysis (DEA) based on the definition of efficiency as the ratio of outputs produced by the unit and inputs spent in the production process. Standard data envelopment analysis models divide the units into inefficient and efficient ones. The efficient units receive the efficiency score $100 \%$ by standard DEA models and can be further classified by so called super-efficiency DEA models. The paper discusses the possibility of using the AHP model with interval pairwise comparisons for evaluation and classification of efficient units and compares given1results with super-efficiency DEA scores. The proposed approach is applied in assessing the efficiency of pension funds in the Czech Republic - the results given by super-efficiency DEA models and by the interval AHP model are compared and discussed.

\section{Introduction}

The importance of pension funds is very high in current economic conditions in the Czech Republic. It is given by several factors. Not very positive demographic perspectives of the Czech population belong among them to the one of the most significant ones. Very low birth rates together with higher average population life length have negative impact on the pension system. That is why in this situation the responsibility of each individual is increasing and the state supports the individuals investing into pension funds. Since 1998 there were established together 12 pension funds. All of them offer standard services of long-term saving of money bringing usually the above-average rate of return comparing to other low risk investment instruments. The investing into the pension funds is characterized by the following:

- it is a saving instrument with a very long time horizon based on monthly saving of very low amounts leading to a significant target amount when the pension age is reached,

- without investing into the pension funds the life standard of the most individuals can be significantly lower after they reach the pension age,

- the higher rate of return together with fixed monthly state support makes the investing into the pension funds one of the most economic and at the same time the safest investment opportunities.

Currently, 12 pension funds secured by the state control operate on the pension fund market in the Czech Republic. The aim of the paper to analyze the current situation in this field and try to estimate the efficiency of pension funds in order to offer to interested persons a recommendation for their investment decisions. The standard modeling tool for evaluation of efficiency is data envelopment analysis (DEA). The standard DEA models split the evaluated units into the efficient and inefficient ones and they are not able to differentiate among the efficient ones. The classification of efficient units can be done by so called super-efficiency DEA models. Our aim is to develop an AHP model for evaluation of pension funds (generally production units) indicated as efficient ones by means of a DEA model used in the first stage of the analysis and compare the results given by super-efficiency DEA models with conclusions from the AHP model. 
The paper is organized as follows. The next section contains a brief description of basic DEA models including the super-efficiency models used in our analysis. Section 3 is a brief introduction to AHP models with interval comparisons. Section 4 formulates an IAHP model for evaluation of efficiency of decision making units and discusses numerical results of both DEA and IAHP analysis. The last section contains summarization of the paper and discussion about the future research.

\section{Data envelopment analysis models}

Data envelopment analysis (DEA) is a tool for measuring the relative efficiency and comparison of decision making units (DMU). The DMUs are usually described by several inputs that are spent for production of several outputs. Let us consider the set $\mathrm{E}$ of $n$ decision making units $\mathrm{E}=\left\{\mathrm{DMU}_{1}, \mathrm{DMU}_{2}\right.$, $\left.\ldots, \mathrm{DMU}_{\mathrm{n}}\right\}$. Each of the units produces $r$ outputs and for their production $m$ inputs are spent. Let us denote $\mathbf{x}^{\mathrm{j}}=\left\{\mathrm{x}_{\mathrm{ij}}, \mathrm{i}=1,2, \ldots, m\right\}$ the vector of inputs and $\mathbf{y}^{\mathrm{j}}=\left\{\mathrm{y}_{\mathrm{ij}}, \mathrm{i}=1,2, \ldots, r\right\}$ the vector of outputs of the $\mathrm{DMU}_{\mathrm{j}}$. Then $\mathbf{X}$ is the $(m, n)$ matrix of inputs and $\mathbf{Y}$ the $(r, n)$ matrix of outputs. The basic principle of the DEA in evaluation of efficiency of the $\mathrm{DMU}_{\mathrm{q}}, \mathrm{q} \in\{1,2 .,,, . \mathrm{n}\}$ consists in looking for a virtual unit with inputs and outputs defined as the weighted sum of inputs and outputs of the other units in the decision set - $\mathbf{X} \boldsymbol{\lambda}$ a $\mathbf{Y} \boldsymbol{\lambda}$, where $\lambda=\left(\lambda_{1}, \lambda_{2}, \ldots, \lambda_{\mathrm{n}}\right), \boldsymbol{\lambda}>\mathbf{0}$ is the vector of weights of the DMUs. The virtual unit should be better (or at least not worse) than the analyzed unit $\mathrm{DMU}_{\mathrm{q}}$. The problem of looking for a virtual unit can be generally formulated as a standard linear programming problem:

minimize

subject to

$$
\begin{aligned}
& \mathrm{z}=\theta-\varepsilon\left(\mathbf{e}^{\mathbf{T}} \mathbf{s}^{+}+\mathbf{e}^{\mathbf{T}} \mathbf{s}^{-}\right), \\
& \mathbf{Y} \lambda-\mathbf{s}^{+}=\mathbf{y}^{\mathbf{q}}, \\
& \mathbf{X} \lambda+\mathbf{s}^{-}=\theta \mathbf{x}^{\mathbf{q}} \\
& \lambda, \mathbf{s}^{+}, \mathbf{s}^{-} \geq \mathbf{0}
\end{aligned}
$$

where $\mathrm{e}^{\mathrm{T}}=(1,1, \ldots, 1)$ and $\varepsilon$ is a infinitesimal constant (usually $\left.10^{-8}\right)$. The variables $\mathrm{s}^{+}, \mathrm{s}^{-}$are just slack variables expressing the difference between virtual inputs/outputs and appropriate inputs/outputs of the $\mathrm{DMU}_{\mathrm{q}}$. Obviously, the virtual inputs/outputs can be computed using the optimal values of variables of the model (1) as follows:

$$
\begin{aligned}
& \mathbf{x}^{\mathbf{q},}=\mathbf{x}^{\mathbf{q}} \theta^{*}-\mathbf{s}^{-}, \\
& \mathbf{y}^{\mathbf{q}}=\mathbf{y}^{\mathbf{q}}+\mathbf{s}^{+} .
\end{aligned}
$$

The $\mathrm{DMU}_{\mathrm{q}}$ is to be considered as efficient if the virtual unit is identical with evaluated unit (does not exist a virtual unit with better inputs and outputs). In this case $\mathbf{Y} \lambda=\mathbf{y}^{\mathbf{q}}, \mathbf{X} \boldsymbol{\lambda}=\mathbf{x}^{\mathbf{q}}$ and the minimum value of the objective function $\mathrm{z}=1$. Otherwise the $\mathrm{DMU}_{\mathrm{q}}$ is not efficient and minimum value of $\theta<1$ can be interpreted as the need of proportional reduction of inputs in order to reach the efficient frontier. The presented model is input oriented model because its objective is to find a reduction rate of inputs in order to reach the efficiency. The output oriented models maximize the expansion rate of outputs in order to reach the efficient frontier. The mathematical formulation of output oriented model can be as follows:

$\begin{array}{ll}\text { maximize } & \mathbf{z}=\phi+\varepsilon\left(\mathbf{e}^{\mathbf{T}} \mathbf{s}^{+}+\mathbf{e}^{\mathbf{T}} \mathbf{s}^{-}\right), \\ \text {subject to } & \mathbf{Y} \lambda-\mathbf{s}^{+}=\phi \mathbf{y}^{\mathbf{q}}, \\ & \mathbf{X} \boldsymbol{\lambda}+\mathbf{s}^{-}=\mathbf{x}^{\mathbf{q}}, \\ & \lambda, \mathbf{s}^{+}, \mathbf{s}^{-} \geq \mathbf{0} .\end{array}$

In model (2) the evaluated unit $\mathrm{DMU}_{\mathrm{q}}$ is efficient if the optimal objective value $\mathrm{z}=1$, ie. $\phi=1$ and all the slack variables equal to zero. The optimal objective function for inefficient units is greater than 1.

The models (1) and (2) suppose constant returns to scale - it is considered that a percentual change of inputs leads to the same percentual change of outputs. The modification of the above models taking into account variable return to scale is derived from them by adding the convexity constraint $\mathbf{e}^{\mathrm{T}} \lambda=1$. The efficiency score in standard DEA models is limited to unit (100\%). Nevertheless, the number of efficient units identified by DEA models and reaching the maximum efficiency score $100 \%$ can be relatively high 
and especially in problems with a small number of decision units the efficient set can contain almost all the units. In such cases it is very important to have a tool for a diversification and classification of efficient units. That is why several DEA models for classification of efficient units were formulated. In these models the efficient scores of inefficient units remain lower than $100 \%$ but the efficient score for efficient units can be higher than $100 \%$. Thus the efficient score can be taken as a basis for a complete ranking of efficient units. The DEA models that relax the condition for unit efficiency are called superefficiency models.

The super-efficiency models are always based on removing the evaluated efficient unit from the set of units. This removal leads to the modification of the efficient frontier and the super-efficiency is measured as a distance between evaluated unit and a unit on the new efficient frontier. Of course several distance measures can be used - this leads to different super-efficiency definitions. The first super-efficiency DEA model was formulated in (Andersen and Petersen, 1993). Its input oriented formulation (3) below is very close to the standard input oriented formulation of model (1). In this model the weight $\lambda_{\mathrm{q}}$ of the evaluated unit $\mathrm{DMU}_{\mathrm{q}}$ is equated to zero. This cannot influence the efficient score of the inefficient units but the efficient score of the efficient units is not limited by $100 \%$ in this case. The input oriented formulation of the Andersen and Petersen model with constant return to scale is as follows:

minimize

$$
\begin{aligned}
& \mathrm{z}=\theta, \\
& \sum_{\mathrm{j}=1, \neq \mathrm{q}}^{\mathrm{n}} \mathrm{x}_{\mathrm{ij}} \lambda_{\mathrm{j}}+\mathrm{s}_{\mathrm{i}}^{-}=\theta \mathrm{x}_{\mathrm{iq}}, \quad \mathrm{i}=1,2, \ldots, \mathrm{m}, \\
& \sum_{\mathrm{j}=1, \neq \mathrm{q}}^{\mathrm{n}} \mathrm{y}_{\mathrm{ij}} \lambda_{\mathrm{j}}-\mathrm{s}_{\mathrm{i}}^{+}=\mathrm{y}_{\mathrm{iq}}, \quad \mathrm{i}=1,2, \ldots, \mathrm{r}, \\
& \lambda, \mathrm{s}^{+}, \mathrm{s}^{-} \geq 0 .
\end{aligned}
$$

The output oriented super-efficiency model with constant or variable returns to scale can be formulated similarly to previous models.

\section{The interval AHP model}

The Analytic Hierarchy Process (AHP) is a powerful tool for analysis of complex decision problems. The AHP organizes the decision problem as a hierarchical structure containing always several levels. The first (topmost) level defines a main goal of the decision problem and the last (lowest) level describes usually the decision alternatives or scenarios. The levels between the first and the last level can contain secondary goals, criteria and subcriteria of the decision problem. The number of the levels is not limited, but in the typical case it does not exceed four or five. Let us consider a simple three-level hierarchy that can represent a standard decision problem with finite set of alternatives - evaluation of n-alternatives $\mathrm{X}_{1}, \mathrm{X}_{2}$, $\ldots, X_{n}$, by k-criteria $Y_{1}, Y_{2}, \ldots, Y_{k}$, (Figure 1). The decision maker expresses his preferences or compares importance of the elements on the given level with respect to the element of the preceding level. The information resulting from decision maker's judgements in the given level of the hierarchy is synthesised onto the local priorities. They can express, e.g. relative importance of criteria (weight coefficients - in Figure 1 denoted by $v_{j}, i=1,2, \ldots, k$ ) or preference indices of the units with respect to the given criterion $\left(w_{i j}, i=1,2 \ldots, n, j=1,2, \ldots, k\right)$. In the standard AHP model the decision maker judgements are organised into paired comparison matrices at each level of the hierarchy. The judgements are point estimates of the preference between two elements of the level. Let us denote the paired comparison matrix $\mathbf{A}=\left\{\mathrm{a}_{\mathrm{ij}} \mid \mathrm{a}_{\mathrm{ji}}=\right.$ $\left.1 / a_{i j}, a_{i j}>0, i, j=1,2, \ldots, k\right\}$, where $k$ is the number of elements of the particular level. Saaty (1990) proposes to use for preference expression $\mathrm{a}_{\mathrm{ij}}$ integers in the range 1 through 9 , where 1 means that the $\mathrm{i}$-th and the $\mathrm{j}$ th element are equally important and 9 means that the i-th element is absolutely more important than the j-th element. The local priorities are derived by solving the following eigenvector problem 


$$
\begin{aligned}
& \text { A.v }=\lambda_{\text {max }} \mathbf{v}, \\
& \sum_{i=1}^{k} v_{i}=1,
\end{aligned}
$$

where $\lambda_{\max }$ is the largest eigenvalue of $\mathbf{A}$ and $\mathbf{v}$ is the normalised right eigenvector belonging to $\lambda_{\max }$.

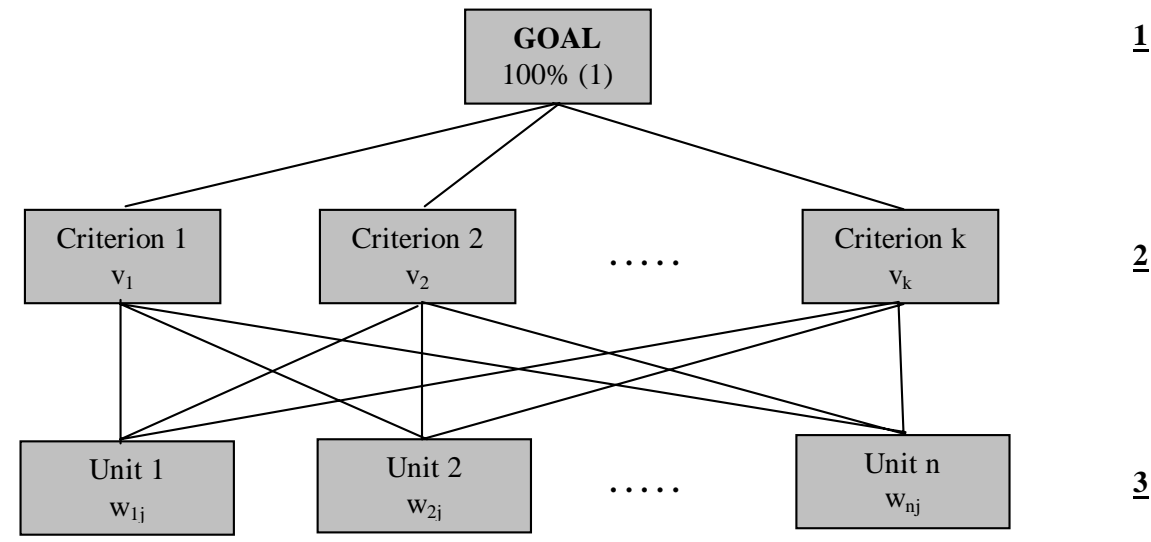

Figure 1: Three-level hierarchy.

In the standard deterministic AHP approach the decision maker always specifies point estimates that express his preference relations between two elements in the given hierarchical level. It can often be very difficult to fulfil this condition for decision makers. They feel much better and closer to have the possibility to express their preferences as interval estimates. For instance, instead of giving that the i-th element is four times as preferable as the j-th element, he can assert that the i-th element is at least two but no more than five times as preferable as the j-th element.

The AHP model with interval decision maker judgements is usually called interval AHP (IAHP) model. It is characterised by interval pairwise comparison matrices given as follows:

$$
\mathrm{A}=\left[\begin{array}{cccc}
1 & <\mathrm{p}_{12}, \mathrm{q}_{12}> & \cdots & <\mathrm{p}_{1 \mathrm{k}}, \mathrm{q}_{1 \mathrm{k}}> \\
<\mathrm{p}_{21}, \mathrm{q}_{21}> & 1 & \cdots & <\mathrm{p}_{2 \mathrm{k}}, \mathrm{q}_{2 \mathrm{k}}> \\
\vdots & \vdots & \cdots & \vdots \\
<\mathrm{p}_{\mathrm{k} 1}, \mathrm{q}_{\mathrm{k} 1}> & <\mathrm{p}_{\mathrm{k} 2}, \mathrm{q}_{\mathrm{k} 2}> & \cdots & 1
\end{array}\right]
$$

where $\mathrm{p}_{\mathrm{ij}}$ is lower bound and $\mathrm{q}_{\mathrm{ij}}$ upper bound for preference relation $\left(\mathrm{a}_{\mathrm{ij}}\right)$ between the $\mathrm{i}$-th and $\mathrm{j}$-th element. Due to the reciprocal nature of the pairwise comparison matrices the relation $\mathrm{p}_{\mathrm{ij}} \cdot \mathrm{q}_{\mathrm{ji}}=1$ holds for all $\mathrm{i}, \mathrm{j}=1,2, \ldots, \mathrm{k}$.

The judgements in the IAHP can be considered as random variables defined over the given interval. In this way the IAHP changes from the deterministic model to the model with some stochastic features. That is why it cannot be analysed in the traditional way - by solving the eigenvector problem (4). It is necessary to look for new approaches that will respect stochastic features. The random variables for description of interval judgements can be selected from the available probabilistic distributions. We will use the uniform distribution defined over the interval $\left\langle\mathrm{p}_{\mathrm{ij}}, \mathrm{q}_{\mathrm{ijj}}\right\rangle$ and in our numerical experiments below. The preferences of elements derived form from matrix A are random variables. Their characteristics can be computed by several approaches - we used a Monte Carlo simulation that is very simple and offers lower and upper bounds for the preferences in a very short time. 


\section{DEA analysis versus IAHP model}

The DEA models are based on maximization of the individual efficiency of the evaluated unit under the constraints that the efficiency of all the other units is lower than $1(100 \%)$. In this formulation the variables of the model are the weights of the inputs and outputs, i.e. weights of the criteria in the sense of multiple criteria decision making. The DEA model finds the best weights in order to maximize the efficiency of the evaluated unit $\mathrm{DMU}_{\mathrm{q}}$. In standard DEA models the efficient units receive the efficient score $1(100 \%)$. With regard to the number of units of the evaluated set on one hand and the number of inputs and outputs on the other hand, the number of efficient units can be relatively high. That is why several super-efficiency models were proposed in order to make it possible to classify the efficient units. In the super-efficiency models the units indicated originally as efficient become the super-efficiency measure greater than one. Than, this measure can be used for their discrimination and classification. Our aim was to compare the results given by super-efficiency DEA models with multiple criteria decision making methodology that can be represented very well by an AHP model. We used an IAHP model because it makes it possible to incorporate to the analysis an appropriate level of uncertainty that is typical for economic decision making problems.

The discussion concerning both the approaches will be demonstrated on a small numerical example with real economic background. It is the problem of evaluation of efficiency of available pension funds in the Czech Republic. We worked with the data set for 12 pension funds, each of them was characterized by the following seven criteria (the data are from year 2003):

- INP 1 - the number of customers [thousands],

- INP 2 - total assets [mil. CZK],

- INP 3 - equity capital [mil. CZK],

- INP 4 - total costs [mil. CZK],

- OUT 1 - appreciation of the customer deposits for the last year (2003) [\%],

- OUT 2 - average appreciation of the customer deposits for the last three years (2001 - 2003) [\%],

- OUT 3 - net profit [mil. CZK].

For DEA analysis, first four criteria were taken as inputs and the remaining ones as outputs of the model. The criterion matrix is given in Table 1.

\begin{tabular}{|l|r|r|r|r|r|r|r|}
\hline & \#of cust. & assets & equity & tot. costs & appr. 1 & appr. 3 & profit \\
\hline Allianz & 106 & 4095 & 77.0 & 49.5 & 3.00 & 3.69 & 1.29 \\
\hline Credit Suisse & 611 & 22592 & 549.0 & 454.1 & 3.36 & 3.67 & 5.22 \\
\hline CSOB Progres & 18 & 452 & 56.0 & 15.1 & 4.30 & 4.15 & 1.13 \\
\hline CSOB Stabilita & 304 & 8508 & 298.6 & 203.3 & 2.30 & 2.83 & 10.87 \\
\hline Generali & 23 & 789 & 74.0 & 15.5 & 3.00 & 3.90 & 0.45 \\
\hline ING PF & 346 & 9767 & 289.1 & 221.7 & 4.00 & 4.27 & 0.26 \\
\hline CP PF I & 225 & 6348 & 290.7 & 184.7 & 3.34 & 3.65 & 6.83 \\
\hline CP PF II & 518 & 12441 & 522.5 & 297.3 & 3.10 & 3.37 & 6.90 \\
\hline CS PF & 401 & 10954 & 223.5 & 238.8 & 2.64 & 3.31 & 1.10 \\
\hline KB PF & 285 & 11776 & 441.6 & 166.0 & 3.40 & 4.14 & 6.40 \\
\hline PF Ostrava & 19 & 935 & 71.0 & 18.2 & 2.44 & 2.68 & 0.04 \\
\hline PF Zemsky & 14 & 468 & 87.9 & 23.2 & 4.01 & 4.24 & 2.03 \\
\hline
\end{tabular}

Table 1: Pension funds - criterion matrix.

As it is possible to see, the funds listed in the previous table are of different nature. Four of them are very small (CSOB Progres, Generali, PF Ostrava and PF Zemsky) and the remaining ones are significantly bigger. That is why we decided to analyze them separately. In Table 2, there are results of DEA analysis of both groups of funds (large and small). We used the basic envelopment DEA model with variable returns to scale with output orientation and the super-efficiency model under the same assumptions. First column of Table 2 contains efficiency scores of the evaluated units - the presented score is a reciprocal 
value of the optimal score given by the model because in output oriented models the score of inefficient units is greater than one. Our transformation can be better explained - higher score corresponds to more efficient unit. The same holds for super-efficiency scores presented in the last column of Table 2. Of course the super-efficiency scores are available for units indicated as efficient by the standard model only. The word "infeasible" for super-efficiency score of Allianz fund means that the corresponding VRS super-efficiency model has no feasible solution. This situation can occur in VRS super-efficiency models very often and in this case it disables the possibility to classify the efficient units.

\begin{tabular}{|l|r|l|}
\hline DEA/VRS model & $\begin{array}{l}\text { efficiency } \\
\text { score }\end{array}$ & $\begin{array}{l}\text { super-eff } \\
\text { score }\end{array}$ \\
\hline Large funds & & \\
\hline Allianz & 100.00 & infeasible \\
\hline Credit Suisse & 96.12 & \\
\hline CSOB Stabilita & 100.00 & 159.24 \\
\hline ING PF & 100.00 & 119.87 \\
\hline CP PF I & 100.00 & 122.40 \\
\hline CP PF II & 95.64 & \\
\hline CS PF & 82.14 & \\
\hline KB PF & 100.00 & 112.40 \\
\hline Small funds & & \\
\hline CSOB Progres & 100.00 & infeasible \\
\hline Generali & 93.72 & \\
\hline PF Ostrava & 64.06 & \\
\hline PF Zemsky & 100.00 & infeasible \\
\hline
\end{tabular}

Table 2: Efficiency measures given by DEA models.

The IAHP model for evaluation of efficiency is very simple and it is presented on Figure 2. In the model $\mathrm{q}_{1}$ is the total weight of inputs and $\mathrm{q}_{2}$ is the total weight of outputs, $\mathrm{q}_{1}+\mathrm{q}_{2}=1 . \mathrm{qi}_{\mathrm{j}}, \mathrm{j}=1,2, \ldots, \mathrm{m}$, are the weights of single inputs and $\mathrm{qo}_{\mathrm{j}}, \mathrm{j}=1,2, \ldots \mathrm{r}$, are the weights of single outputs. Preference indices $\mathrm{u}_{\mathrm{ij}}$, $\mathrm{i}=1,2, \ldots, \mathrm{n}, \mathrm{j}=1,2, \ldots, \mathrm{m}+\mathrm{r}$, express the preference of the $\mathrm{i}$-th alternative (pension fund) with respect to the j-th input/output.

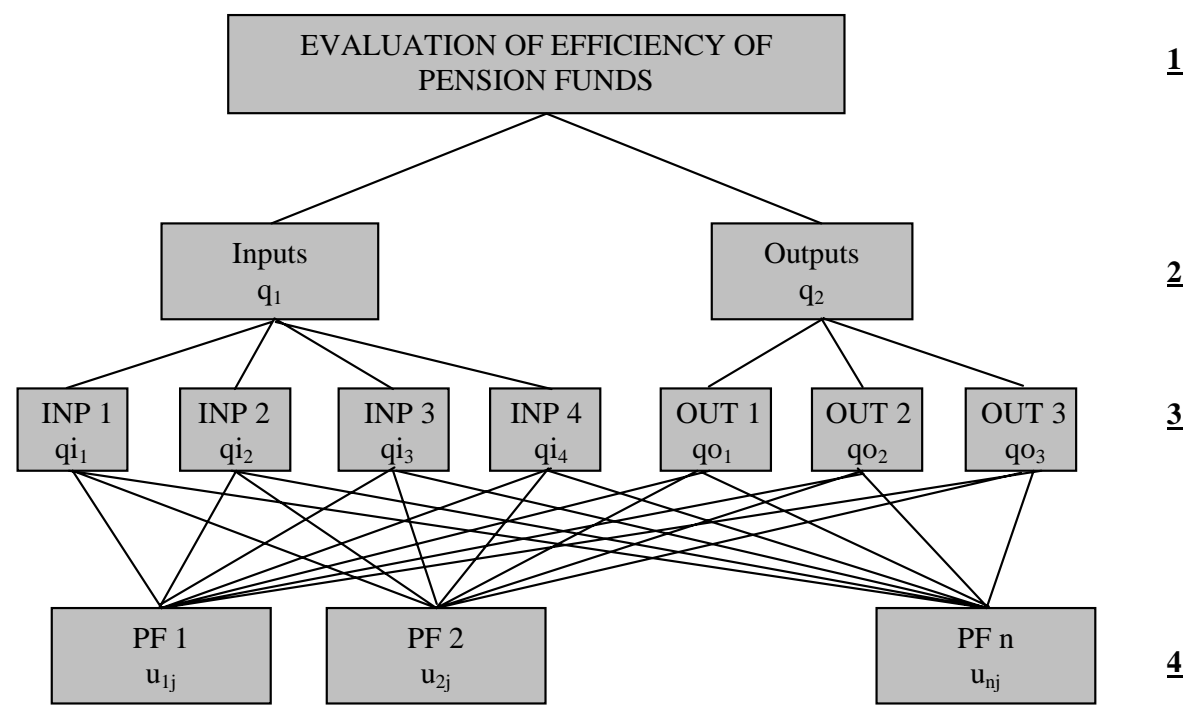

Figure 2: AHP model for evaluation of efficiency. 
The global preference indices of alternatives $\mathrm{p}_{\mathrm{i}}, \mathrm{i}=1,2, \ldots, \mathrm{n}$, are synthesized from previous hierarchical levels as follows:

$$
p_{i}=\sum_{j=1}^{m+r} u_{i j}, i=1,2, \ldots, n
$$

In our analysis the weights of the inputs and outputs were not derived by the AHP model but they were either to set up directly as constants or optimized as variables of proposed simulation model. In the model the pairwise comparisons of alternatives (pension funds) with respect to all inputs and outputs were given as random variables with uniform distribution defined on the interval $<a, b>$. The comparisons reflect given criterion values but by this way it is possible to use different returns to scale for different inputs and outputs. It is one of the advantages of this approach. Our numerical experiments were realized on the set of five big pension funds identified as efficient by DEA model with variable returns to scale. The pairwise comparisons of all alternatives with respect to first input (number of employees) and output (appreciation of customer deposits) are given in the following table (free cells are the reciprocal values):

\begin{tabular}{|l|r|r|r|r|r|}
\hline & \multicolumn{6}{|l|}{ Number of customers } \\
\cline { 2 - 6 } & Allianz & CSOB & ING & CP PF & KB PF \\
\hline Allianz & 1 & $<6,8>$ & $<7,9>$ & $<3,5>$ & $<5,7>$ \\
\hline CSOB & & 1 & $<1,2>$ & & \\
\hline ING & & & 1 & & \\
\hline CP PF & & $<1,3>$ & $<3,5>$ & 1 & $<2,3>$ \\
\hline KB PF & & $<1,2>$ & $<2,4>$ & & 1 \\
\hline
\end{tabular}

\begin{tabular}{|r|r|l|r|r|}
\hline \multicolumn{5}{|c|}{ Appreciation of customer deposits } \\
\hline Allianz & CSOB & ING & CP PF & KB PF \\
\hline 1 & $<3,5>$ & & & \\
\hline & 1 & & & \\
\hline$<4,6>$ & $<5,7>$ & 1 & $<3,5>$ & $<3,5>$ \\
\hline$<1,3>$ & $<3,5>$ & & 1 & 1 \\
\hline$<1,3>$ & $<3,5>$ & & & 1 \\
\hline
\end{tabular}

Table 3: Pairwise comparisons of the IAHP model.

The comparison matrices for the remaining inputs and outputs are given in the similar way.

The DEA models maximize the individual efficiency of evaluated units by looking for optimum weights of inputs and outputs. That is why we did not work with weights derived by the IAHP model but we tried to optimize the weights in order to reach the best efficiency score of the evaluated alternative. We used the following requirements to the set of the weights:

1. The sum of weights equals to 1 , all the weights have to be greater than 0.05 (AHP I).

2. The sum of weights of inputs equals to 0.5 , the same holds for the weights of outputs. All the weights have to be greater than 0.05 (AHP II).

3. All the weights are fixed to value $1 /(\mathrm{m}+\mathrm{r})$, ie. $1 / 7$ in our example (AHP III).

\begin{tabular}{|l|r|r|r|r|}
\hline Pension funds & $\begin{array}{c}\text { DEA/VRS } \\
\text { super-eff. }\end{array}$ & AHP I & AHP II & AHP III \\
\hline Allianz & infeasible & 0.4574 & 0.3262 & 0.3334 \\
\hline CSOB Stabilita & 159.24 & 0.3608 & 0.2583 & 0.1449 \\
\hline ING PF & 119.87 & 0.3652 & 0.2764 & 0.1894 \\
\hline CP PF & 122.40 & 0.2123 & 0.2056 & 0.1699 \\
\hline KB PF & 112.40 & 0.2397 & 0.2138 & 0.1625 \\
\hline
\end{tabular}

Table 4: Comparison of super-efficiency measures.

The optimization run was realized by means of Crystal Ball which is an MS Excel add-in application for Monte Carlo experiments. Crystal Ball contains a special tool for optimization under stochastic conditions called OptQuest. This tool can find optimum values of variables (weights of inputs and outputs in our case) in stochastic environment that can be modeled within MS Excel. The optimization criterion is the efficiency score $\mathrm{p}_{\mathrm{i}}, \mathrm{i}=1,2, \ldots \mathrm{n}$, of the evaluated alternative that is to be maximized. Because the efficiency score under our stochastic conditions is a random variable we tried to maximize its mean value. We always used a five minutes optimization run for all the alternatives with 100 trials per one simulation. The results are presented in Table 4 . The first column of Table 4 contains super-efficiency scores computed by 
the Andersen and Petersen DEA model with variable returns to scale, the remaining three columns contain maximized efficiency score of pension funds given by the presented IAHP model with different weight constraints (weight sets I, II and III).

The DEA/VRS model is not able to evaluate the Allianz fund, the best among the others is the CSOB Stabilita fund and the worse is the KB pension fund. The results given by the IAHP models are quite different. If we consider the same weights for all the inputs and outputs (AHP III), the Allianz fund is classified on the top and the remaining funds are very close each other. The results for the set of weights AHP I and AHP II are almost identical. The best is the Allianz fund, the mean efficiency score of the CSOB and ING pension funds is more or less the same and on the bottom of the ranking are the last two funds. The optimal weights computed by the OptQuest application for the set of weight constraints are presented in Table 5.

\begin{tabular}{|l|r|r|r|r|r|}
\hline & Allianz & CSOB & ING PF & CP PF & KB PF \\
\hline \# of customers & 0.2574 & 0.0509 & 0.0500 & 0.0500 & 0.0500 \\
\hline total assets & 0.1116 & 0.0500 & 0.0500 & 0.7000 & 0.0500 \\
\hline equity & 0.3266 & 0.0500 & 0.0500 & 0.0500 & 0.0500 \\
\hline total costs & 0.1545 & 0.0500 & 0.0500 & 0.0500 & 0.0500 \\
\hline appreciation 1 & 0.0500 & 0.0500 & 0.4592 & 0.0500 & 0.0500 \\
\hline appreciation 3 & 0.0500 & 0.0510 & 0.2908 & 0.0500 & 0.5676 \\
\hline profit & 0.0500 & 0.6981 & 0.0500 & 0.0500 & 0.1824 \\
\hline
\end{tabular}

Table 5: Optimal weights for the set AHP I.

\section{Conclusions}

The aim of the paper was to verify how the AHP models can be used for efficiency evaluation of production units and compare the results given by proposed interval AHP model with efficiency scores computed by DEA models. In contrary to super-efficiency DEA models the advantage of the IAHP approach consists in several points:

- the IAHP model can use different scales for different inputs and outputs according to the decision maker preferences,

- in super-efficiency DEA models with VRS not all the units receive their super-efficiency score (the problems need not be always feasible),

- the IAHP model can deal with categorial inputs and outputs without any transformation,

- the IAHP model offers a possibility of sensitivity analysis of results with respect to the inputs and/or outputs.

The main disadvantage of the AHP (IAHP) models comparing to the DEA models consists in preparing of data for the analysis (pairwise comparison matrices) and in time consuming length of the optimization analysis. Nevertheless, by using the AHP model the decision maker can receive new information useful for the global analysis of the efficiency of the evaluated set of units.

Future research in this field will be focused on comparison other super-efficiency DEA models (slack based models) with IAHP models and other multiple criteria decision making techniques. Several real-life economic applications will deal as background for numerical experiments.

\section{Acknowledgements}

The research is partially supported by the Grant Agency of the Czech Republic - project no. 402/03/1360. 


\section{References}

Andersen, P. and Petersen, N.C. (1993) "A procedure for ranking efficient units in data envelopment analysis”, Management Science, 39, 1261-1264.

Cooper, W.W., Seiford L.M. and Tone, K. (2000) Data Envelopment Analysis, Boston, Kluwer Publ.

Fiala, P. and Jablonsky, J. (2002) Models for Performance Evaluation of Central European Firms, Research report of the project "Productivity gap", IWH Halle, http://www.iwhhalle.de/projects/productivity-gap.

Jablonsky, J. (2004) "Models for Evaluation of Efficiency of Production Units (in Czech)” Politicka ekonomie, 2, pp.206-220.

Saaty, T.L. (1990) The Analytic Hierarchy Process, Pittsburgh, RWS Publications.

Saaty, T.L. (1996) Decision making with Dependence and Feedback: The Analytic Network Process, Pittsburgh, RWS Publications.

Zhu, J. (2003) Quantitative Models for Performance Evaluation and Benchmarking. Boston, Kluwer Publ. 